\title{
Autoeficacia Musical Y Ansiedad Escénica: Variables Relacionadas En Músicos En Formación
}

\author{
Luis Manuel Cuartero Oliveros \\ Francisco Javier Zarza Alzugaray \\ José Elías Robles Rubio \\ Óscar Casanova López \\ Universidad de Zaragoza, Spain
}

doi: 10.19044/esj.2017.v13n25p1 URL:http://dx.doi.org/10.19044/esj.2017.v13n25p1

\begin{abstract}
The explanatory capacity of self-efficacy on achievement has made it one of the main psychological constructs on the field of educational research. Perceptions of self-efficacy become more relevant, if possible, in musical education whose purpose is professional practice and in the end public performance. Music performance anxiety, for its part, has been studied in numerous investigations, finding itself responsible for academic failure and even dropping out the musical career. Both constructs are related and influence the attainment of achievement. This study reveals the relationship between these two constructs, obtaining inversely proportional correlations between the factors musical self-efficacy for performing and vulnerability and stage anxiety cognitions of music performance anxiety; delving into the knowledge of both constructs are also observed differences according to sex. The results obtained provide the necessary evidence to deepen the knowledge of the variables that lead to musical achievement.
\end{abstract}

Keywords: Music education, self-efficacy, music performance anxiety, musical success

\section{Resumen}

La capacidad explicativa de la autoeficacia sobre el logro le ha permitido convertirse en uno de los constructos psicológicos principales en el campo de la investigación educativa. Las percepciones de autoeficacia se tornan más relevantes, si cabe, en las enseñanzas musicales cuyo fin es el ejercicio profesional y en definitiva la interpretación en público. Por su parte, la ansiedad escénica ha sido objeto de estudio en numerosas investigaciones, encontrándose como responsable del fracaso académico e incluso del abandono de la carrera musical. Ambos constructos están relacionados e 
influyen en la consecución del logro. Este estudio pone de manifiesto la relación entre estos dos constructos, obteniendo correlaciones inversamente proporcionales entre los factores interpretación de autoeficacia musical y los factores indefensión y cogniciones de ansiedad escénica; ahondando en el análisis de ambos constructos se observan, además, diferencias en función del sexo. Los resultados obtenidos proporcionan la evidencia necesaria para profundizar en el conocimiento de las variables que conducen al logro musical.

Palabras clave: Educación musical, autoeficacia, ansiedad escénica, logro musical

\section{Introducción}

Durante las últimas décadas la ansiedad escénica, entre otros, se ha postulado como un factor muy a tener en cuenta en la consecución del logro musical. Sin embargo, desde su introducción dentro del marco de la teoría de socio-cognitiva (Bandura, 1977) y tras ser considerado un elemento esencial para alcanzar el éxito en cualquier disciplina, el constructo psicológico denominado autoeficacia ha suscitado gran interés en el contexto de la investigación educativa.

Este logro debe ser entendido como la consecución de determinadas metas; un objetivo general, una motivación bien intrínseca o extrínseca que anima a emprender conductas específicas. Sin embargo, conocer dichas metas o el mejor medio para alcanzarlas no es suficiente, la percepción acerca de la propia eficacia se alza como requisito fundamental para desarrollar con éxito las acciones conducentes al logro de los objetivos personales (Bandura, 1977). No basta con ser capaz, hay que juzgarse capaz (Blanco, Martínez, Zueck y Gastelúm, 2011).

La autoeficacia, específica y dinámica, debe ser entendida como la creencia de una persona sobre su capacidad de tener éxito en una situación determinada; la propia capacidad para organizar y ejecutar las acciones necesarias para manejar situaciones futuras (Bandura, 1977). Ejerce una profunda influencia en la forma de pensar, de comportarse y de sentir de cada individuo determinando, junto con otras variables, en el éxito de las propias acciones (Carbonero y Merino, 2008).

Concretamente, podría decirse que en el proceso de alcanzar el éxito musical, las características personales o las habilidades musicales entre otras (Freeman, 1991), son importantes, pero la autoeficacia emerge como un factor relevante; constructo psicológico entendido como el elemento que media entre las habilidades objetivas de cada individuo, su ejecución y sus consecuencias. Es por ello evidente la importancia de las percepciones de autoeficacia en las enseñanzas musicales en las que el estudiante debe 
adquirir una serie de competencias complejas relacionadas con su aprendizaje autónomo y la interpretación en público (Zimmerman, 2002).

La autoeficacia debe ser diferenciada de otros constructos cercanos como el autoconcepto tanto en especificidad como en contenido (Schunk y Pajares, 2001) ya que, mientras este último hace referencia a percepciones de competencia en general, la autoeficacia se ocupa de creencias personales sobre capacidad en tareas específicas. Ambos constructos contribuyen a la motivación y al logro académico (Shunk y Pajares, 2001), sin embargo, la autoeficacia demuestra mayor poder predictivo (McPherson y McCormick, 2006). La autoeficacia es un constructo psicológico dinámico (Berry y West, 1993), cualidad que la ha convertido en un tema de gran interés en el ámbito de la investigación educativa.

Autores como Usher y Pajares (2006) identificaron una relación positiva entre autoeficacia y logro en tareas relacionadas con la lectura, y Lopez y Lent (1992) encontraron resultados similares en matemáticas.

La importancia y relevancia de las creencias de autoeficacia se hace más evidente, si cabe, en las enseñanzas musicales cuyo fin es el ejercicio profesional. Estas enseñanzas demandan una serie de competencias complejas que pueden ser influidas por las percepciones de autoeficacia. El aprendizaje autorregulado, el esfuerzo físico, mental y emocional necesarios para el estudio de un instrumento o la capacidad de interpretación en público son factores necesarios pero no garantizan alcanzar el éxito musical (McPherson y McCormick, 2006; Zarza, 2014).

Ya en la década de los setenta, Greenberg (1970) presentó evidencia de la influencia de la autopercepción sobre el rendimiento y las actitudes de la música de los estudiantes. Pero no será hasta el siglo XXI cuando aparecen investigaciones relevantes en esta línea (Zelenak, 2011).

Según McCormick y McPherson (2003 y 2006), en un contexto de exámenes y/o actuaciones musicales, la autoeficacia y la actuación real muestran una fuerte relación, siendo ésta un buen predictor de la actuación pública. La autoeficacia presenta, además, una importancia real en las actividades que requieren actuaciones públicas (Zimmerman, 2000), especialmente cuando se producen en contextos en los que tanto las personas que actúan como las actuaciones en sí mismas están sometidas a procesos de evaluación externa.

Sin embargo, y a pesar de lo dicho hasta el momento, considerar la autoeficacia como aquella variable determinante que corrige todos los problemas sería un error. No existe un factor único y exclusivo, siendo la suma de todos ellos, por pequeña que sea su aportación, la que permita determinar la ecuación del logro musical.

Entre los otros factores a tener en cuenta se encuentra la ya mencionada ansiedad escénica. Entendida como la experiencia de aprensión 
angustiosa que puede llegar a disminuir las habilidades interpretativas en un contexto público hasta un grado que no garantiza la aptitud musical del individuo, el entrenamiento o el nivel de preparación de éste (Salmon, 1990). La ansiedad escénica es considerada uno de los grandes problemas que afecta de una u otra forma a músicos de todo el mundo (Yoshie, Kudo, Murakoshi y Ohtsuki, 2009).

Según Barlow (2000), la explicación teórica de dicha ansiedad encuentra explicación en la interacción de tres factores: vulnerabilidad biológica, psicológica general y específica. Los dos primeros, junto con las características personales y contextuales participan en la presencia de unos niveles de indefensión $\mathrm{u}$ otros, sin embargo, parece ser necesaria la interacción de los dos factores de vulnerabilidad anteriores con un tercer factor, denominado vulnerabilidad específica, para el desarrollo de un trastorno de ansiedad específico (Barlow, 2000). La sensibilidad al castigo, el pesimismo, el optimismo y la autoeficacia son otros constructos a los que Barlow hace referencia en este sentido y que pueden influir en la génesis final del antedicho problema.

Así, partiendo del modelo de Barlow, Kenny (2009a y 2009b) desarrolla un marco teórico complejo propio del contexto musical explicando la ansiedad escénica como el fruto de la interacción de tres factores: contexto, indefensión y cogniciones específicas.

En lo relativo a variables de carácter personal, el sexo se ha mostrado como una de las variables que más capacidad explicativa de las diferencias presenta. En este sentido no son pocas las investigaciones (Kenny, 2011; Papageorgi, Hallam y Welch, 2007; Zarza, Casanova y Orejudo, 2016a) que reportan de manera sistemática que las mujeres presentan mayores niveles de indefensión y ansiedad escénica que los hombres. Sin embargo, en el caso de la autoeficacia encontramos la opción inversa, es decir, son los hombres los que reportan mayores niveles de autoeficacia que las mujeres tal y como apunta Zelenak (2011).

La consecuencia de dichas percepciones de ansiedad escénica son observables a nivel cognitivo, fisiológico y conductual. Son comunes los pensamientos negativos previos a la actuación o las pérdidas de memoria así como temblores en extremidades, boca seca o manos sudorosas. El escape o la evitación y el consumo de sustancias pueden ser otras repercusiones que en casos extremos pueden conducir al abandono de la formación e incluso de la carrera musical (Zarza et al., 2016a).

De todo lo anterior se desprende la necesidad de profundizar en el conocimiento de ambos constructos siendo el propósito principal de este estudio analizar los niveles de autoeficacia musical y ansiedad escénica percibida en estudiantes de música en conservatorio en el contexto español. En este sentido, se analizará la posible relación entre ambas variables con el 
fin de aportar mayor evidencia en el estudio de los factores que conducen al logro musical.

\section{Método}

\section{Participantes}

La muestra está formada por 119 estudiantes de Conservatorios Superiores de Música de la Comunidad Valenciana de los cuales el 59.7\% son hombres y el 40.3\% mujeres. La edad media de la muestra es 22.41 años (D. T. $=3.834$ ) comprendida entre 18 y 46 años. La muestra comprende la mayoría de las especialidades instrumentales y se distribuye de la siguiente manera: bombardino $(n=4)$, canto $(n=1)$, clarinete $(n=19)$, contrabajo $(n=$ $2)$, fagot $(n=2)$, flauta $(n=7)$, guitarra $(n=3)$, musicología $(n=1)$, oboe $(n$ $=9)$, percusión $(n=3)$, piano $(n=6)$, saxofón $(n=6)$, trombón $(n=9)$, trompa $(n=14)$, trompeta $(n=15)$, tuba $(n=3)$, viola $(n=3)$, violín $(n=6)$ y violoncello $(n=6)$.

\section{Materiales}

La recogida de datos se ha llevado a cabo mediante un dosier de cuestionarios que incluye una batería de preguntas relacionadas con datos socio-demográficos y pedagógicos así como los cuestionarios de autoeficacia musical para el aprendizaje y la interpretación de Ritchie y Williamon (2007) adaptados y validados para la población española por Cuartero (2016). Por último, se incluye el cuestionario de ansiedad escénica KMPAI (Kenny, Davis y Oates, 2004) en su versión española (Zarza, Orejudo, Casanova y Mazas, 2016b).

La primera sección está dedicada a datos socio-demográficos y pedagógicos. Instrumento, edad, curso, edad de comienzo, relación de los padres con la música o el tipo y cantidad de conciertos realizados son algunas de las variables introducidas en el diseño debido a su influencia, contrastada empíricamente, sobre los constructos objeto de estudio (Papageorgi et al., 2007; Zelenak, 2010).

La autoeficacia se analiza mediante una escala tipo Likert 1-7 (desacuerdo-acuerdo) formada por 22 ítems agrupados en dos sub-escalas: autoeficacia musical para el aprendizaje y autoeficacia musical para la interpretación. Esta versión presenta un muy buena consistencia interna para cada uno de sus factores: aprendizaje $(\alpha=.812)$ e interpretación $(\alpha=.837)$.

Por su parte, el KMPAI comprende 26 ítems medidos con una escala tipo Likert de 7 puntos en la que mayores puntuaciones indican mayores niveles de ansiedad. Estos 26 ítems son agrupados en 3 sub-escalas o factores: indefensión $(\alpha=.786)$, cogniciones $(\alpha=.868)$ y contexto $(\alpha=$ .568). 


\section{Procedimiento}

Tras recibir respuesta afirmativa de todos los conservatorios para participar en el estudio, se procedió a la recogida a través de los cuestionarios anteriormente citados vía internet. La imposibilidad de acceder a toda la población de estudiantes de conservatorio españoles afectó al tipo de muestreo, optando por el criterio de disponibilidad, tanto del centro como del alumnado.

El análisis de datos se desarrolló siguiendo las indicaciones de Pardo y Ruiz (2005), utilizado el software estadístico SPSS versión 22.0, paquete informático que permite realizar procedimientos estadísticos habituales en el tratamiento de datos de tipo cuantitativo. Análisis descriptivo y relaciones entre variables, comparación de medias mediante técnicas de análisis de varianza y análisis correlaciónales son la técnicas utilizadas para obtener los resultados que se presentan a continuación.

\section{Resultados}

Un primer acercamiento a la distribución de ambas escalas llevado a cabo mediante la prueba Kolmogorov-Smirnov permite contrastar la hipótesis nula de normalidad y concluir que tanto los factores aprendizaje (K. $\mathrm{S} .=.968)$ e interpretación $(\mathrm{K} . \mathrm{S} .=.700)$ de la variable autoeficacia como los factores indefensión $(\mathrm{K} . \mathrm{S} .=.495)$, cogniciones $(\mathrm{K} . \mathrm{S} .=.535)$ y contexto $(\mathrm{K}$. $\mathrm{S} .=1.330)$ de ansiedad escénica se ajustan a una distribución normal ( $\mathrm{p}>$ $.05)$.

El factor de aprendizaje presenta una media $(\mathrm{m}=58.395 ; \mathrm{D} . \mathrm{T} .=$ 9.668) significativamente superior $(\mathrm{t}=6.511 ; \mathrm{p}=.000)$ al factor interpretación $(\mathrm{m}=53.647$; D. T. $=11.210)$. Concretamente, en el factor aprendizaje un $18.5 \%$ de los encuestados se sitúan más alejados de una desviación típica por debajo de la media. Este porcentaje sube hasta un $20.2 \%$ en el factor interpretación.

La media real $(m=41.908 ;$ D. T. $=13.833)$ del factor cogniciones no es significativamente distinta $(\mathrm{t}=-1.650 ; \mathrm{p}=.102)$ a la media teórica del instrumento, 44. Por su parte, el factor indefensión presenta una media de 34.874 (D. T. $=9.420$ ) y el factor contexto obtiene una media de 8.941 (D. T. $=3.540)$.

En la variable ansiedad escénica, el factor indefensión obtiene un $18.5 \%$ de encuestados con puntuaciones por encima de la desviación típica de la media real. Un 34\% de los estudiantes puntúan de la misma forma en el factor cogniciones, mientras que un $24.4 \%$ hacen lo propio en el factor contexto.

Existen correlaciones significativas y directamente proporcionales entre los factores aprendizaje e interpretación de autoeficacia musical $(\mathrm{r}=$ 
$.719 ; \mathrm{p}=.000)$ y entre los factores indefensión y cogniciones de la variable ansiedad escénica $(r=.558 ; \mathrm{p}=.000)$ (Tabla 1$)$.

Dicha correlación significativa se da de forma inversamente proporcional entre los factores aprendizaje e indefensión $(\mathrm{r}=-.345 ; \mathrm{p}=$ $.000)$, aprendizaje y cogniciones $(\mathrm{r}=-.519 ; \mathrm{p}=.000)$, interpretación $\mathrm{e}$ indefensión $(\mathrm{r}=-.439 ; \mathrm{p}=.000)$, interpretación y cogniciones $(\mathrm{r}=-.732 ; \mathrm{p}=$ .000). Por su parte, el factor contexto de la variable ansiedad escénica no correlaciona con ninguno de los factores incluidos en el análisis.

Tabla 1. Correlación entre factores

\begin{tabular}{cccccc}
\hline & AEF Apren & AEF Inter & AE Inde & AE Cogni & AE Con \\
\hline AEF Apren & 1 & $.719^{* *}$ & $-.345^{* *}$ & $-.519^{* *}$ & -.046 \\
AEF Inter & $.719^{* *}$ & 1 & $-.439^{* *}$ & $-.732^{* *}$ & -.033 \\
AE Inde & $-.345^{* *}$ & $-.439^{* *}$ & 1 & $.558^{* *}$ & .009 \\
AE Cogni & $-.519^{* *}$ & $-.732^{* *}$ & $.558^{* *}$ & 1 & -.055 \\
AE Con & -.046 & -.033 & .009 & -.055 & 1 \\
\hline
\end{tabular}

** La correlación es significativa en el nivel 0.01 ( 2 colas).

Por último, se ha realizado un análisis de varianza que permite comparar los grupos definidos por la variable sexo en cada uno de los factores anteriormente citados. El contraste de Levene ha permitido contrastar la homogeneidad de varianzas en los factores objeto de estudio ( $\mathrm{p}$ $>$.05) concluyendo que, en las poblaciones definidas por la variable sexo, las varianzas de los factores aprendizaje e interpretación de autoeficacia musical y los factores indefensión, cogniciones y contexto de ansiedad escénica son iguales. Por ello, se ha procedido a realizar la comparación de medias utilizando el estadístico ANOVA.

Tal y como se observa en la Tabla 2, se han encontrado diferencias de medias significativas entre hombres y mujeres en los factores interpretación $(\mathrm{F}=5.170 ; \mathrm{p}=.025)$, indefensión $(\mathrm{F}=10.341 ; \mathrm{p}=.002), \mathrm{y}$ cogniciones $(\mathrm{F}=$ $15.938 ; \mathrm{p}=.000)$. El tamaño del efecto del sexo sobre las diferencias de medias obtenidas debe ser considerado como moderado para los factores indefensión $(8.1 \%)$ y cogniciones $(12 \%)$ de ansiedad escénica, y pequeño para el factor interpretación $(4.2 \%)$ de autoeficacia musical según los criterios de Cohen (1988). 
Tabla 2. ANOVA

\begin{tabular}{|c|c|c|c|c|c|c|c|}
\hline & & $\mathrm{N}$ & Media & Des. Est. & $\mathrm{F}$ & Sig. & $\mathrm{h}^{2}$ \\
\hline \multirow{2}{*}{ AEF Apren } & Hombre & 71 & 59.169 & 9.07 & \multirow{2}{*}{1.129} & \multirow{2}{*}{.290} & \multirow{2}{*}{.010} \\
\hline & Mujer & 48 & 57.250 & 10.485 & & & \\
\hline \multirow{2}{*}{ AEF Inter } & Hombre & 71 & 55.535 & 11.251 & \multirow{2}{*}{5.170} & \multirow{2}{*}{.025} & \multirow{2}{*}{.042} \\
\hline & Mujer & 48 & 50.854 & 10.659 & & & \\
\hline \multirow{2}{*}{ AE Inde } & Hombre & 71 & 32.676 & 9.151 & \multirow{2}{*}{10.341} & \multirow{2}{*}{.002} & \multirow{2}{*}{.081} \\
\hline & Mujer & 48 & 38.125 & 8.943 & & & \\
\hline \multirow{2}{*}{ AE Cogni } & Hombre & 71 & 37.986 & 13.56 & \multirow{2}{*}{15.938} & \multirow{2}{*}{.000} & \multirow{2}{*}{.120} \\
\hline & Mujer & 48 & 47.708 & 12.206 & & & \\
\hline \multirow{2}{*}{ AE Con } & Hombre & 71 & 9.169 & 3.513 & \multirow{2}{*}{.728} & \multirow{2}{*}{.395} & \multirow{2}{*}{.006} \\
\hline & Mujer & 48 & 8.604 & 3.589 & & & \\
\hline
\end{tabular}

\section{Discusión y conclusiones}

Los resultados obtenidos muestran diferencias de medias en los constructos psicológicos autoeficacia musical y ansiedad escénica, responsables del desarrollo y evolución de los estudiantes de música y por tanto susceptibles de ser estudiados en profundidad.

Tal y como indica Bandura (1977) la autoeficacia es un constructo específico y así lo demuestran las diferencias de medias significativas $(\mathrm{t}=$ $6.511 ; \mathrm{p}=.000)$ obtenidas entre los factores autoeficacia para el aprendizaje $(\mathrm{m}=58.395 ;$ D. T. $=9.668)$ y autoeficacia para la interpretación $(\mathrm{m}=$ 53.647; D. T. $=11.210$ ), siendo este último el que presenta menor puntación contrastando los resultados obtenidos por Ritchie y Williamon (2011). Estos datos reflejan, además, la posible influencia de la ansiedad escénica en actividades que requieren interpretación en público y/o evaluación.

Se ha observado que en torno al $20 \%$ de estudiantes encuestados (18.5\% factor aprendizaje y $20.2 \%$ factor interpretación) presentan puntuaciones inferiores más alejadas de la media, conformando éstos una posible población sobre la que realizar una determinada intervención o cuando menos ser susceptibles de mayor estudio.

Tal y como ocurre en Ritchie y Williamon (2011) los factores de aprendizaje e interpretación correlacionan significativamente $(\mathrm{r}=.719 ; \mathrm{p}=$ .000). Pese a no haber encontrado investigaciones al respecto, es importante subrayar las correlaciones significativas e inversamente proporcionales encontradas entre los factores interpretación de autoeficacia musical y los factores indefensión y cogniciones de ansiedad escénica. Esta correlación pone de manifiesto la influencia de ambos constructos.

Las conclusiones aquí presentadas han de tomarse con precaución debido al tamaño y criterio de elección, disponibilidad, de la muestra. Bien es cierto que dicha muestra puede ser considerada representativa ya que 
contempla la mayoría de las especialidades instrumentales, sin embargo, se considera necesario replicar el trabajo aquí presentado con una muestra más grande y que contemple diferentes niveles formativos en la población española. Se espera que los resultados obtenidos reviertan en la práctica educativa en forma de nuevas propuestas formativas cuyo último fin sea una mejora en los programas de cualificación de los futuros profesionales de la música.

\section{References:}

1. Bandura, A. (1977). Self-efficacy: Toward a unifying theory of behavioral change. Psycological Review, 84(2), 191-215.

2. Barlow, D.H. (2000). Unraveling the mysteries of anxiety and its disorders from the perspective of emotion theory. American Psychologist, 55(11), 1247-1263. doi:10.1037/0003066X.55.11.1247

3. Berry, J.M., \& West, R. L. (1993). Cognitive self-efficacy in relation to personal mastery and goal setting across the lifespan. International Journal of Behavioral Development, 16, 351-379.

4. Blanco, H., Martínez, M., Zueck, M.A., \& Gastélum, G. (2011). Análisis psicométrico de la escala autoeficacia en conductas académicas en universitarios de primer ingreso. Actualidades Investigativas en Educación, 11(3), 1-27.

5. Carbonero, M.A., \& Merino, E. (2008). Autoeficacia y madurez vocacional. Psicothema, 16(2), 229-234.

6. Cohen, J. (1988). Statistical Power Analysis for the Behavioral Sciences (2nd Ed.). Hillsdale, NJ: Erlbaum.

7. Cuartero, L.M. (2016). Autoeficacia Musical y Apoyo Social en estudiantes de conservatorio: Adaptación de dos cuestionarios y estudio preliminar. Trabajo Fin de Máster. Zaragoza: Universidad de Zaragoza.

8. Freeman, J. (1991). Gifted children grown up. London: Heinemann Educational.

9. Greenberg, M. (1970). Musical achievement and the self-concept. Journal of Research in Music Education, 18(1), 57-64.

10. Kenny, D. T. (2009a). The factor structure of the revised Kenny Music Performance Anxiety Inventory. In A. Williamon, S. Pretty \& R. Buck (Eds.), Proceedings of the International Symposium on Performance Science 2009 (pp. 37-41). Utrecht, The Netherlands: Association Européenne des Conservatoires (AEC).

11. Kenny, D.T. (2009b). The role of negative emotions in performance anxiety. In P. Juslin \& J. Sloboda (Eds.), Handbook of music and 
emotion: Theory, research, applications (pp. 425-452). Oxford, UK: Oxford University Press.

12. Kenny, D.T. (2011). The psychology of music performance anxiety. Oxford, UK: Oxford University Press.

13. Kenny, D.T., Davis, P., \& Oates, J. (2004). Music performance anxiety and occupational stress amongst opera chorus artists and their relationship with state and trait anxiety and perfectionism. Journal of Anxiety Disorders, 18(6), 757-777. doi:10.1016/j.janxdis.2003.09.004

14. Lopez, F.G., \& Lent, R.W. (1992). Sources of mathematics selfefficacy in high school students. Career Development Quarterly, 4l(1), 3-9.

15. McCormick, J., \& McPherson, G.E. (2003). The role of self-efficacy in a musical performance examination: An exploratory structural equation analysis. Psychology of Music, 31(1), 37-51. doi:10.1177/0305735603031001322

16. McPherson, G.E., \& McCormick, J. (2006). Self-efficacy and music performance. Psychology of Music, 34(3), 322-336. doi:10.1177/0305735606064841

17. Papageorgi, I., Hallam, S., \& Welch, G. (2007). A conceptual framework for understanding musical performance anxiety. Research Studies in Music Education, 28, 83-107. doi:10.1177/1321103X070280010207

18. Pardo, A., \& Ruiz, M.A. (2005). Análisis de datos con SPSS 13 base. Madrid: McGraw Hill.

19. Ritchie, L., \& Williamon, A. (2007). Measuring self-efficacy in music. In A. Williamon \& D. Coimbra (Eds.), Proceedings of the International Symposium on Performance Science 2007 (pp. 307312). Utrecht, The Netherlands: Association Européenne des Conservatoires (AEC).

20. Ritchie, L., \& Williamon, A. (2011). Measuring distinct types of musical self-efficacy. Psychology of Music, 39(3), 328-344. doi:10.1177/0305735610374895

21. Salmon, P. (1990). A psychological perspective on Musical Performance Anxiety: A review of the literature. Medical Problems of Performing Artists, 5(1), 1-11.

22. Schunk, D.H., \& Pajares, F. (2001). The development of academic self-efficacy. In A. Wigfield \& J. S. Eccles (Eds.), Development of achievement motivation (pp. 15-31), San Diego, CA: Academic Press.

23. Usher, E.L., \& Pajares, F. (2006). Sources of academic and selfregulatory efficacy beliefs of entering middle school students. Contemporary Educational Psychology, 31, 125-141. 
24. Yoshie, M., Kudo, K., Murakoshi, T., \& Ohtsuki, T. (2009). Music performance anxiety in skilled pianists: Effects of social-evaluative performance situation on subjective, autonomic, and electromyographic reactions. Experimental Brain Research, 199(2), 117-126. doi:10.1007/s00221-009-1979-y

25. Zarza, F.J. (2014). Variables psicológicas y pedagógicas como predictoras de la ansiedad escénica en estudiantes de Grado Superior de Música de España. Tesis doctoral. Zaragoza: Universidad de Zaragoza. Disponible en https://zaguan.unizar.es/record/47413/files/TESIS-2016-021.pdf

26. Zarza, F.J., Casanova, O. \& Orejudo, S. (2016a). Ansiedad escénica y constructos psicológicos relacionados. Estudiantes de cinco conservatorios superiores de música españoles. RIEM. Revista Internacional de Educación Musical, 4, 13-24. doi:10.12967/RIEM2016-4-p013-024

27. Zarza, F.J., Orejudo, S., Casanova, O., \& Mazas, B. (2016b). Kenny Music Performance Anxiety Inventory: Confirmatory factor analysis of the Spanish version. Psychology of Music, 44(3), 340-352. doi:10.1177/0305735614567932

28. Zelenak, M.S. (2010). Development and Validation of the Music Performance Self-Efficacy Scale. MERI. Music Education Research International, 4, 31-43.

29. Zelenak, M.S. (2011). Self-Efficacy in Music Performance: Measuring the Sources Among Secondary School Music Students. Tesis doctoral sin publicar. Disponible en: http://scholarcommons.usf.edu/etd/3419

30. Zimmerman, B.J. (2000). Self-Efficacy: An essential motive to learn. Contemporary Educational Psychology, 25(1), 82-91. doi:10.1006/ceps.1999.1016

31. Zimmerman, B.J. (2002). Achieving self-regulation: The trial and triumph of adolescence. In F. Pajares \& T. Urdan (Eds.), Academic motivation of adolescents (pp. 1-28). Connecticut: Information Age Publishing. 